\title{
Comparative study of herringbone and stacked-cup carbon nanofibers
}

Yoong-Ahm Kim*, Takuya Hayashi, Satoru Naokawa, Takashi Yanagisawa and Morinobu Endo

Faculty of Engineering, Shinshu University, 4-17-1 Wakasato, Nagano-shi 380-8553, Japan

Keywords: A. Carbon nanofibers; B. Chemical vapor deposition; C. Transmission electron microscopy; D. Texture

*Corresponding author: Tel: +81-26-269-5212, Fax: +81-26-269-5208, Email: yak@endomoribu.shinshu-u.ac.jp

Due to their small size and their extraordinary physicochemical properties [1-3], much attention has been paid to the interesting $s p^{2}$-based fibrous carbons, including carbon nanotubes. It is generally accepted that carbon nanotubes consist of single or multiple graphene sheets rolled into concentric cylinders: thus giving rise to single wall carbon nanotubes (SWNTs) or multi-walled carbon nanotubes (MWNTs) [4, 5]. Recently, we described a new type of carbon nanofiber, "stacked-cup carbon nanofiber", which exhibited a unique morphology of stacked, truncated conical graphene layers (cups) along the fiber length [6-8].

Previously, three different geometries of carbon nanofibers (e.g., platelet, 
herringbone (or fish-bone), and tubular) were extensively investigated from the early 1970s to the present [9-16]. One of recently prominent study of platelet-type carbon nanofibers using a scanning tunneling microscope (STM) in combined with high resolution transmission electron microscope (HRTEM) have proved that platelet fibers contains a nano-sized building blocks, or nano-rods [15].

Transmission electron microscope (TEM) technique has been considered to be one of the most powerful tools for characterizing the microstructure of carbon nanofibers. But, we should be very careful in obtaining structural information from TEM pictures, since each image is actually a two-dimensional projection. For example, consider the very similar TEM images which were obtained from stacked-cup and herringbone carbon nanofibers (see Figure 1), which has often led to misunderstanding of the microstructure of these catalytically grown carbon fibers.

In this study, by comparing stacked-cup and herringbone carbon nanofibers, we will clarify the differences in microstructure (or texture) utilizing the published experimental data and calculations (e.g., synthesis conditions, the status of metal particles, macro-morphology etc.) [10, 14-16]. For any application, exact understanding of fiber geometry is essential in order to control the properties of the carbon nanofibers (e.g., diameter, length, mechanical strength and surface structure (e.g., edge sites)).

Basic common thing of these fibers is that they are generally prepared by a catalytic chemical vapor deposition method (CVD). When compared to the arc-discharge and laser ablation methods, the CVD technique is a very controllable, cost-effective technique. Furthermore, this technique has been adopted as the most useful technique for large-scale production of carbon nanotubes. Basic pre-conditions for synthesizing carbon nanotubes are metal catalyst, carbon precursor and thermal 
energy. Therefore, right combination of these three components makes it possible to selectively synthesize various types of carbon nanotubes, ranging from SWNTs and MWNTs to carbon nanofibers $[6,17,18]$. Most important factor for controlling the growth and therefore the properties of fibrous carbons are the nano-sized metal catalysts [9], which must catalytically decompose the carbon feedstock and provide relatively high carbon solubility. Since nano-sized metal particles with high ratio of the surface atom to internal atom are expected to exhibit high surface energy, area, and mobility, lowered melting point and increased carbon solubility, they consequently exhibit a different metal-carbon phase diagram compared to bulk metal. Thus, understanding the status of the metal particle during fiber growth is highly critical for obtaining geometrical information about the fiber. Empirically, we can infer that the metal particles responsible for the growth of stacked-cup nanofibers are in the molten-state, when considering the size distribution of metal particles (which is roughly related to the diameter distribution of fibrous carbon) with reaction conditions (including temperature and types of carbon feed stock) (see Table 1) [19] whereas those of herringbone nanofibers are in the solid state during fiber growth. Therefore, the diffusion-controlled growth rates of stacked-cup carbon nanofibers are significantly higher than those of herringbone type carbon nanofibers. It is noteworthy that the duration of fiber growth for metal particles in the reaction chamber for the case of stacked-cup carbon nanofiber is as short as 0.3 seconds. Moreover, the abundant metal particles incorporated in the hollow core of carbon nanofibers (see Figure 2) strongly suggest a molten state for the metal particles during fiber growth because of capillary forces [20]. As a result, molten metal particles give rise to straight fibers with a round cross-section (see Figure 3 in ref [8]) and a large hollow core (up to 60nm) (see Figure 2 in ref. [8]). On the other hand, 
considering the kinetic and thermodynamic factors limiting effective CO disproportionation reactions at $500-700^{\circ} \mathrm{C}$ at atmospheric pressure, it is reasonable that metal particles in herringbone carbon nanofibers are in the solid state. This assumption is strongly supported by the fact that the cross-sectional morphology of herringbone type fibers is rectangular, hexagonal and heptagonal rather than circular because the nanofiber growth is considered to be the result of replication of the shape of catalytic metal particle at the tip of the carbon fiber [15].

Differences in geometries between stacked-cup carbon nanofibers and herringbone carbon nanofibers are thus basically derived from their different growth modes due to their molten or solid state of their metal catalyst particles. These result in different surface, physical and chemical properties. Hence, the factors summarized in Table 1 are not independent, but rather interdependent. The relatively rugged surfaces and wavy fibrous morphology of herringbone type carbon nanofiber result in a higher surface area which can be explained by the slow diffusion-controlled growth rate of carbon within the solid metal catalyst. From the geometrical features of both fibers (see Figure 1), it is sure that both fibers exhibit higher active surface area (ASA) [21], which can be defined as accessible and reactive sites (e.g., the edges of the graphene layers) of conventional carbon materials [21]. The lower surface area and ASA (3-5 $\left.\mathrm{m}^{2} / \mathrm{g}\right)$ for stacked-cup nanofiber is attributed to a smooth surface formed by the deposited carbon during nanofiber growth, which is an inevitable consequence of CVD process at higher reaction temperature. Therefore, by selecting the duration, temperature and time judiciously we can control the thickness of the deposited carbon [6] which manifests itself in TEM as small fringes roughly aligned along the fiber length. This layer acts as a protective layer, resulting in improved mechanical properties of single fibers. On the 
other hand, even though herringbone fibers showed two times larger ASA (8-10 m²/g), but is quite low as we expected. Therefore, it is expected that highly reactive edge sites on the outer surfaces of nanofibers are stabilized through the bent morphology, and chemical and physical sorption of water. When considering application of these fibers as filler in various composites, macro-morphology will be the determining factor for dispersion. In this sense, herringbone fibers will require great care during compounding because a compromise between dispersion and shortening phenomena must be reached.

Even though both fibers have two important common aspects: (1) growth by the catalytic chemical vapor deposition method and (2) very similar TEM images (with stacked graphene layers tilted with respect to the fiber axis) (see Figure 2), they have different textures and different physical and chemical properties, finally resulting in different applications. It is noteworthy that the status of metal particles during fiber growth is an essential step for determining microstructure and consequently for applications of these fibrous carbons.

Table 1 Comparative summary of characteristics of stacked-cup and herringbone carbon nanofibers.

\begin{tabular}{lll}
\hline I. D. & Stacked-cup NF & Herringbone type NF \\
\hline Synthetic system & Floating reactant system & Seeding system ${ }^{1)}$ \\
\hline Synthetic temp. & Above $900^{\circ} \mathrm{C}$ & Ca. $600^{\circ} \mathrm{C}$ \\
\hline Reaction time ${ }^{2)}$ & 0.3 second & Above 30 minutes (up to 2hrs) \\
\hline Carbon feedstock & Methane (or natural gas) & Carbon monoxide \\
\hline Growth rate & Very high & Low \\
\hline Diameter ${ }^{3)}$ & $50-150 \mathrm{~nm}$ & $20-100 \mathrm{~nm}(?)$ \\
\hline
\end{tabular}




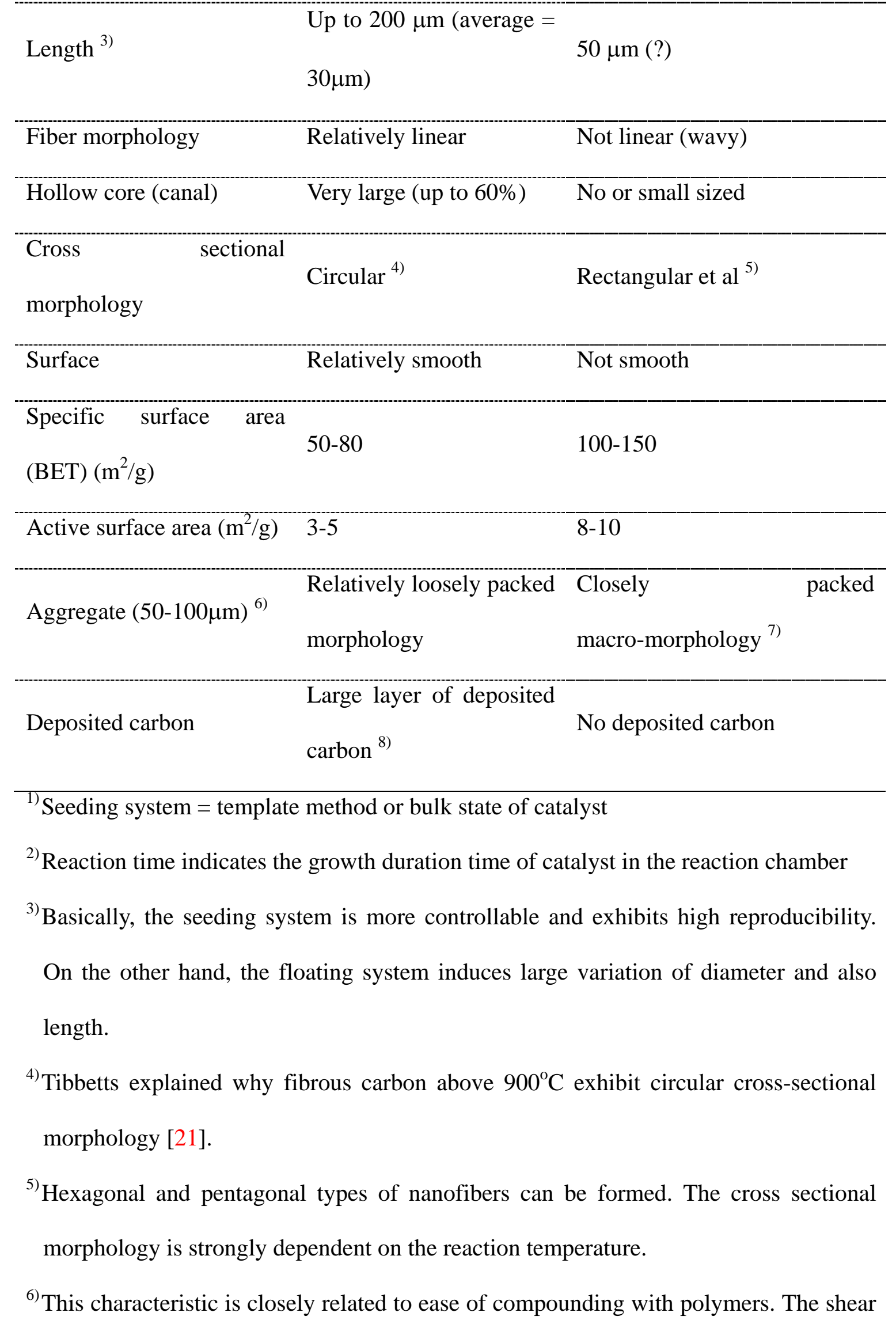


forces for homogeneous dispersion in a polymer will be different. Resultantly, shortening patterns in compounding process will be different.

${ }^{7)}$ Fiber growth in confined space induces closely packed morphology of nanofibers

${ }^{8)}$ The kinetics of carbon deposition (coating or thermal cracking of carbon feedstock) increases abruptly when the reaction temperature is above $900^{\circ} \mathrm{C}$. For the case of stacked-cup carbon nanofibers, this phenomenon is unavoidable. 


\section{References}

[1] Oberlin A, Endo M, Koyama T. Filamentous growth of carbon through benzene decomposition. J. Crystal Growth 1976;32:335-49.

[2] Endo M. Grow carbon fibers in the vapor phase. Chem Tech 1988;18:568-76

[3] Iijima S. Helical microtubles of graphitic carbon. Nature 1991;354:56-8.

[4] Dresselhaus MS, Dresselhaus G, Eklund PC. Science of fullerenes and carbon nanotubes. Academic Press:San Diego. 1996:756-864.

[5] Saito R, Dresselhaus G, Dresselhaus MS. Physical properties of carbon nanotubes. Imperial College Press:Tokyo. 1998:1-251.

[6] Endo M, Kim YA, Hayashi T, Fukai Y, Oshida K, Terrones M, Yanagisawa T, Higaki S, Dresselhaus MS. Structural characterization of stacked cup type nanofibers with an entirely hollow core. Appl Phys Lett 2002;80(7):1267-9.

[7] Endo M, Kim YA, Ezaka M, Osada K, Yanagisawa T, Hayashi T, Terrones M, Dresselhaus MS. Selective and efficient impregnation of metal nanoparticles on cup-stacked-type nanofibers. Nano Letters 2003;3 (6): 723-6.

[8] Endo M, Kim YA, Hayashi T, Yanagisawa T, Muramatsu H, Ezaka M, Terrones H, Terrones M, Dresselhaus MS. Microstructural changes induced in stacked cup carbon nanofibers by heat treatment. Carbon 2003;41:1941-1947.

[9] Baker RTK, Barber MA, Harris PS, Feates FS, Waite RJ. Nucleation and growth of carbon deposits from the nickel:catalyzed decomposition of acetylene. J Catalysis 1972;26:51-62.

[10]Baker RTK. The formation of filamentous carbon. In Walker PL Jr., editor Chemistry and Physics of Carbon, Vol. 14, New York:Marcel Dekker. 1978:84-165.

[11]Baker RTK. Harris PS, Terry S. Unique form of filamentous carbon. Nature 
$1975 ; 253: 37-8$.

[12]Audier M, Oberlin A, Oberlin M, Coulon M, Bonnetain L. Morphology and crystalline order in catalytic carbons. Carbon 1981;19:217-24.

[13] Murayama H, Maeda T. A novel form of filamentous graphite. Nature 1990;345:791-3.

[14]Rodriguez NM, Chambers A, Baker RTK. Catalytic engineering of carbon nanostructures. Langumuir 1995;11:3862-6.

[15] Yoon S-H, Lim S, Hong S-H, Mochida I, An B, Yokogawa K. Carbon nano-rod as a structural unit of carbon nanofibers. Carbon 2004;42(15):3087-95.

[16] Tanaka A, Yoon S-H, Mochida I. Preparation of highly crystalline nanofibers on Fe and $\mathrm{Fe}-\mathrm{Ni}$ catalyst with a variety of graphene plane alignments. Carbon 2004;42(3):591-7.

[17]Endo M, Hayashi T, Kim YA, Muramatu H, Ezaka M, Watts PCP, Nishimura K, Tsukada T. The possible route to large-scale production of SWNTs through combinational techniques of substrate and floating Methods. J Nanosci Nanotech 2004;4(1/2):132-5.

[18]Endo M, Muramatsu H, Hayashi T, Kim YA, Terrones M, Dressehaus MS. Buckypaper from coaxial structure. Nature 2005;433:476.

[19]Homma, Y., Kobayashi Y., Maeda F. Effect of catalysts on carbon nanotube growth on silicon substrate in chemical vapor deposition. J Surf. Sci Soc Jap 2004;25(6): 339-344.

[20]Ajayan PM, Iijima S. Capillarity-induced filling of carbon nanotubes. Nature $1993 ; 361: 333-4$

[21]Leon y Leon CA, Radovic LR. Interfacial chemistry and electrochemistry of carbon 
surfaces. In: Thrower PA, Editor. Chemistry and physics of carbon, vol. 25, New York; Dekker (California, USA); American Carbon Society, 1994;221-2.

[22] Tibbetts GG. Why are carbon filaments tubular? J Crys Growth 1984;66:632-38.

\section{Figure Captions}

Figure 1 (a), (c) Atomic models of stacked-cup and herringbone carbon nanofibers, (b) and (d) their respective TEM simulated images for atomic model (a JEM2000 (TEM simulation), electron energy $=200 \mathrm{kV}$, spherical aberration $=2.7 \mathrm{~mm}$, aperture radius $=$ 0.71/Å, defocus $=-60 \mathrm{~nm}$ ). Both nanofibers exhibited very similar TEM simulated images. Furthermore, stacked-cup carbon nanofibers show a round cross-sectional morphology while herringbone type carbon nanofibers exhibit a rectangular cross-sectional morphology.

Figure 2 (a), (b) HRTEM images of metal particles in the hollow cores of stacked-cup carbon nanofibers. 


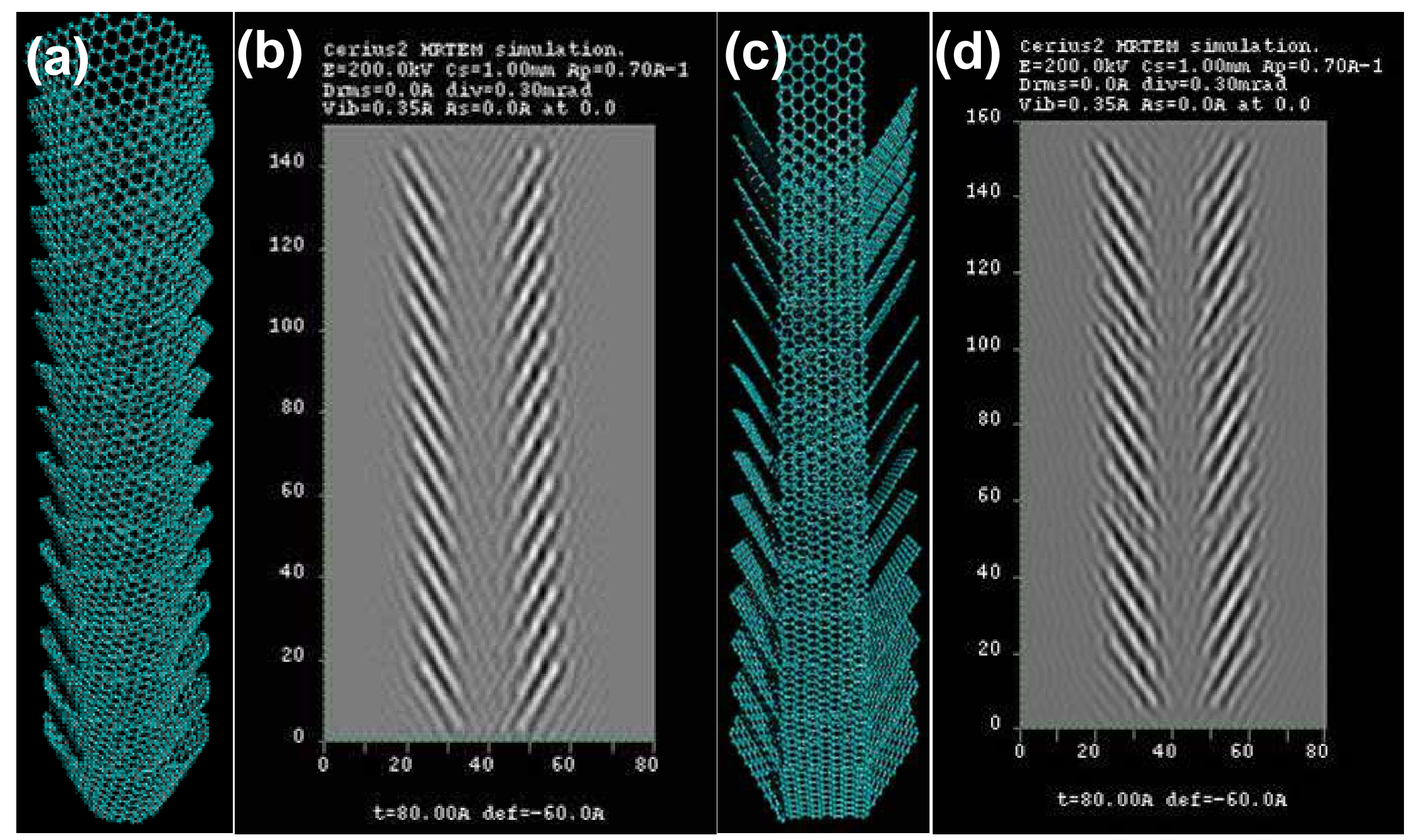

Figure 1 


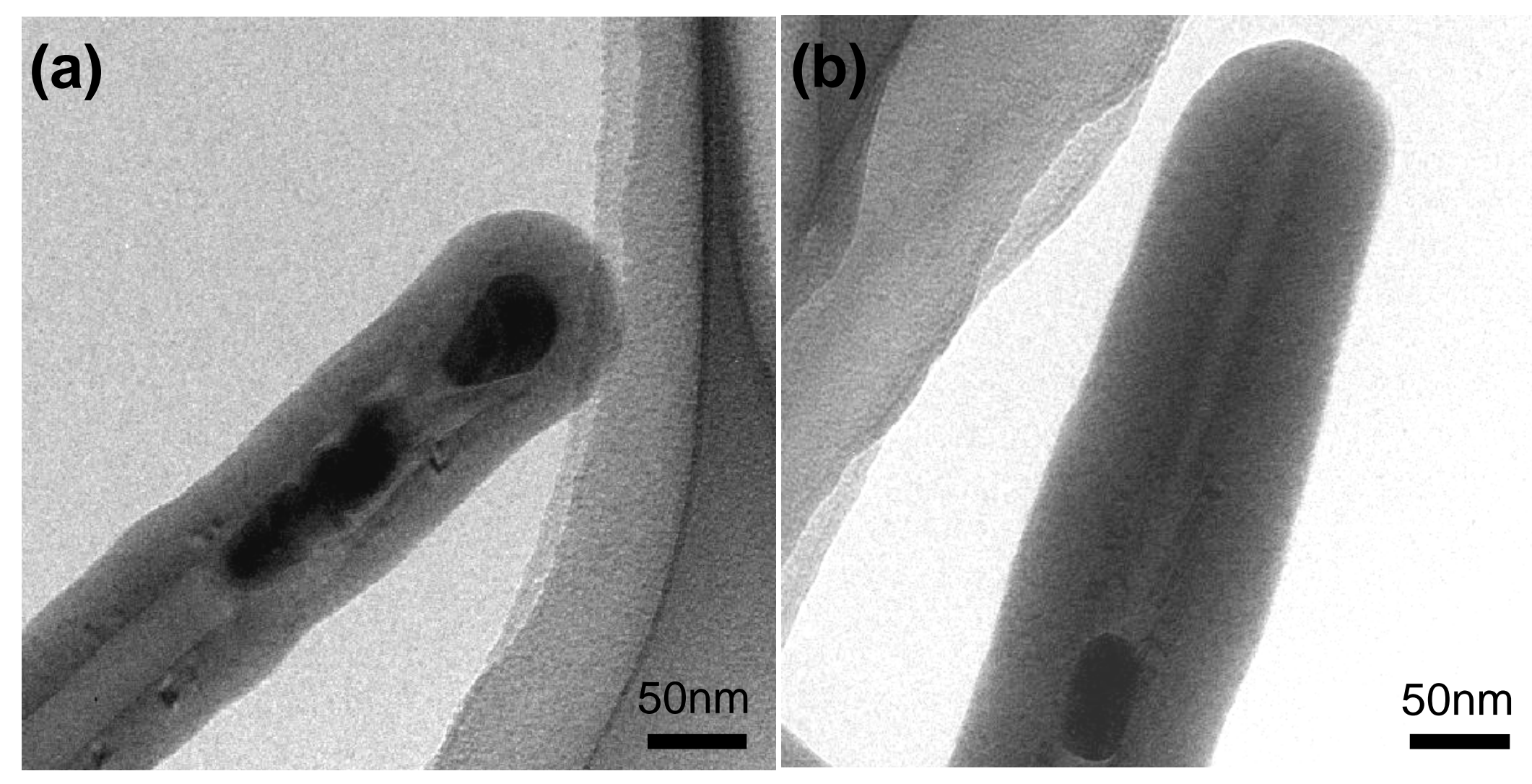

Figure 2 